\title{
In Vitro Fertilization Success and Associated Factors: A Prospective Cohort Study
}

\author{
Azra Azmoudeh ${ }^{1}$, Zahra Shahraki $^{2 * \mathbb{D}}$, Fatemeh-Sadat Hoseini ${ }^{1}$, Firoozeh Akbari-Asbagh ${ }^{1}$, Fatemeh \\ Davari-Tanha ${ }^{3}$, Forough Mortazavi ${ }^{3}$
}

\begin{abstract}
Objectives: In vitro fertilization (IVF)success depends on many factors whose independent roles have not been fully investigated. The aim of this study was to evaluate the role of associated factors in women undergoing IVF in Iran.

Materials and Methods: In a prospective cohort study, 160 women who referred to infertility center of Moheb-Yas hospital for IVF between March 2015 and March 2016 were enrolled. A long ovarian stimulation protocol was administered using a gonadotropinreleasing hormone agonist $(\mathrm{GnRH})$. Anti-Mullerian hormone $(\mathrm{AMH})$, progesterone, estradiol, and endometrial thickness were measured. Two expert embryologists categorized blastocysts as good or poor. Pregnancy was judged by serum human chorionic gonadotropin measurement 14-15 days after embryo transfer.

Results: Mean age of the cases was 32 years and mean body mass index (BMI) was $20 \mathrm{~kg} / \mathrm{m}^{2}$. Clinical pregnancy rate was $20.6 \%$. A significant relationship was found between IVF success and AMH, the number of FSH injections, endometrial thickness, grade of the embryos, and the number of embryos produced. Logistic regression analysis showed that only AMH $>0.6$ was an independent predictor of IVF success (odds ratio $[\mathrm{OR}]=6.22$, CI [2.4-16.2]). Further analysis showed a significant relationship between AMH level and IVF success in women $\leq 35$.

Conclusions: The overall success rate of IVF/ET was $20.6 \%$. AMH is a significant predictor of IVF success and may be an important factor in IVF success in young women.

Keywords: Infertility, In vitro fertilization, Embryo research, Anti-Mullerian hormone, Iran
\end{abstract}

\section{Introduction}

Infertility which is defined as the inability to get pregnant after one year of unprotected sex is affecting near 20\% of couples worldwide and $25 \%$ of couples in developing countries $(1,2)$. Mental, physical, sexual, and social aspects of infertile couples' lives are affected by infertility (3-5), addressing the necessity of proper intervention.

Advances in assisted reproductive technology (ART) for infertile couples has made fast progress since 1976 and researchers have tried to find suitable treatment options for each infertile couple (6). Advances in ART improved the outcomes of in vitro fertilization/embryo transfer (IVF/ET) in recent years; however, the pregnancy rate has not exceeded $40 \%$ per treatment cycle yet (7).

In addition to some demographic factors such as ethnicity, the cause of infertility, age, subfertility duration, parity (8-10), and lifestyle factors (11), oocytes retrieved (9), endometrial thickness (8), the number of embryos transferred (12) and quality of blastocysts (10) are considered as important factors in ART success. Furthermore, elevated basal follicle stimulating hormone (FSH), low number of antral follicles and premature luteinization may negatively affect ART outcomes $(9,13)$.
IVF practices were introduced to Iran in 1987. By now, several centers and clinics have been established and ART is performed widely in Iran (14). Although the role of several factors in IVF success has been investigated in western countries, few studies in Iran examined important factors in IVF success in Iranian women $(4,15-17)$. In addition, no study evaluated the independent effects of the involving factors. We designed this study to evaluate the role of different factors in IVF success in infertility center of Moheb Yas hospital, Iran.

\section{Materials and Methods \\ Study Design, Patient Selection, and Stimulation Protocol This prospective cohort study conducted in the infertility center of Moheb Yas hospital, affiliated to Tehran University of Medical Sciences, between March 2015 and March 2016. The convenient sampling method was used to include 160 infertile women in the study. The inclusion criteria were healthy candidates for IVF/ICSI, and FSH and LH less than 10 which is measured on the third day of the menstrual cycle. The exclusion criteria were a history of endometriosis, ovarian surgery or pelvic radiotherapy, anti-Mullerian hormone (AMH) $<0.05$ measured on}


the third day of the menstrual cycle, endocrinological disorders, and presence of congenital uterine anomalies.

The sample consisted of 160 women who referred to Moheb Yas hospital. A long ovarian stimulation protocol using a GnRH agonist was applied. $0.5 \mathrm{mg}$ buserelin acetate (Superfact, Sanofi, Canada) was administered from day 21 of the cycle. On the first day of menstruation, GnRH dose was reduced by half and FSH ampule, Gonal $\mathrm{F}$ (Serono), was added. All cases underwent trans-vaginal ultrasound examination until $17-18 \mathrm{~mm}$ follicles were observed, and then 10000 IU hCG (Pregnyl, Organon) was injected to stimulate the ovary. Then, the oocyte pick up was performed using a soft catheter under ultrasound guidance and spinal anesthesia. Sperm and oocytes were put together in the lab after oocyte retrieval. In women with a poor history of IVF, the sperm was injected into an oocyte. In the following morning, oocytes were checked for the evidence of fertilization. The embryos were cultured in the IVF lab for 3 days.

Embryo transfer (1-5 embryos) was performed in women with transferable embryos three days after oocyte picking up using a Cook catheter. The number of embryos transferred to the uterus depends on the women's age and the quality of embryos. No more than five embryos were transferred. If more than five good embryos were obtained, they were frozen for further use.

After retrieving oocytes, $100 \mathrm{mg}$ of progesterone per day were injected for three days, then the luteal phase was supported by Cyclogest suppository (Alpharma, Barnstaple, UK), $400 \mathrm{mg}$ for 12 weeks. Pregnancy was assessed by measurement of serum hCG 14-15 days after ET and was then confirmed by ultrasound which visualized at least one gestational sac after two further weeks.

\section{Measurements}

$\mathrm{AMH}$ was measured on day 3 of the menstrual cycle in the endocrinology lab of the hospital. Progesterone and estradiol were measured on the morning of hCG administration. Endometrial thickness was measured on the day of oocyte retrieval by transvaginal ultrasound. Oocytes were retrieved by transvaginal ultrasound- guided aspiration approximately 36 hours after hCG injection. After fertilization of the oocytes with sperms, two physicians categorized blastocysts as good, fair, or poor grade blastocysts, according to the simplified SART embryo scoring system (18).

\section{Statistical Analysis}

SPSS version 22.0 was used for data analysis. Normality of the numeric variables was assessed using ShapiroWilk test. Sample characteristics were determined by means and frequencies. Student $t$ test, ANOVA, and Mann-Whitney $U$ test were used for comparison of the continuous variables. Chi-square and Fisher exact tests were used for comparison of the categorical variables. Logistic regression analysis was applied for odds ratio (OR) calculation. A $P$ value less than 0.05 was considered statistically significant.

\section{Results}

One hundred and sixty women who were referred to Moheb Yas hospital were enrolled in this study. Table 1 shows demographic characteristics of the pregnant and non-pregnant participants. The grade of $91.8 \%$ of embryos was good. Progesterone levels on the day of hCG injection in $76 \%$ of cases were lower than $1 \mathrm{ng} / \mathrm{mL}$. Estradiol levels on the day of hCG injection were higher than $1000 \mathrm{pg} /$ $\mathrm{mL}$ in $77 \%$ of the cases. AMH $\leq 0.6$ was observed in $50.6 \%$ of the cycles. Table 2 shows laboratory characteristics of the pregnant and non-pregnant participants. There was no relationship between IVF success and the levels of progesterone and estradiol, maternal age and body mass index (BMI), and the number of embryos transferred. Maternal age was correlated with the oocyte and embryo number $(\mathrm{r}=-0.16, P=0.05)$.

A significant relationship was found between IVF success and AMH levels, the number of FSH injections, endometrial thickness, embryos grading, and number of the embryos produced. Logistic regression analysis with pregnancy as the dependent variable showed that among $\mathrm{AMH}$, endometrial thickness, number of FSH ampule, embryo grade, and the number of embryo produced, only $\mathrm{AMH}$ is an independent predictor of IVF success $(\mathrm{OR}=$

Table 1. Demographic and Clinical Characteristics

\begin{tabular}{|c|c|c|c|c|c|}
\hline \multirow{2}{*}{ Variable } & \multirow{2}{*}{ Range } & \multirow{2}{*}{$\begin{array}{c}\text { Total } \\
\text { Mean } \pm S D\end{array}$} & \multirow{2}{*}{$\frac{\text { Pregnant }(n=33)}{\text { Mean } \pm S D}$} & \multirow{2}{*}{$\begin{array}{c}\text { Not-pregnant }(\mathrm{n}=127) \\
\text { Mean } \pm S D\end{array}$} & \multirow{2}{*}{$P$} \\
\hline & & & & & \\
\hline Age $(y)^{a}$ & $18-49$ & $33.0 \pm 6.1$ & $33.5 \pm 6.4$ & $32.8 \pm 6.1$ & 0.572 \\
\hline $\mathrm{BMI}\left(\mathrm{kg} / \mathrm{m}^{2}\right)^{\mathrm{b}}$ & $16.2-38.1$ & $25.1 \pm 3.6$ & $25.6 \pm 4.1$ & $24.9 \pm 3.4$ & 0.265 \\
\hline No. of oocyte retrieved ${ }^{b}$ & $1-20$ & $8.0 \pm 7.4$ & $7.4 \pm 4.4$ & $7.5 \pm 3.5$ & 0.680 \\
\hline No. of embryo produced ${ }^{b}$ & $1-14$ & $4.6 \pm 2.7$ & $3.8 \pm 2.5$ & $4.8 \pm 2.8$ & $0.028^{*}$ \\
\hline No. of embryo transferred ${ }^{b}$ & $1-5$ & $2.7 \pm 0.7$ & $2.6 \pm 0.8$ & $2.7 \pm 0.7$ & 0.556 \\
\hline No. of FSH ampule ${ }^{b}$ & $10-72$ & $34.2 \pm 12.2$ & $28.7 \pm 10.2$ & $35.6 \pm 12.3$ & $0.005^{* *}$ \\
\hline Endometrial thickness $(\mathrm{mm})^{\mathrm{b}}$ & $6-33$ & $8.7 \pm 2.3$ & $9.1 \pm 0.9$ & $8.6 \pm 2.5$ & $<0.001 * * *$ \\
\hline
\end{tabular}

${ }^{\mathrm{a}} \mathrm{t}$ test.

${ }^{\mathrm{b}}$ Mann-Whitney $\mathrm{U} ; * P<0.05, * * P<0.01, * * * P<0.001$. 
Table 2. Laboratory Characteristics

\begin{tabular}{|c|c|c|c|}
\hline & Pregnant $(n=33)$ & Not pregnant $(n=127)$ & $P$ \\
\hline $\mathrm{AMH}^{\mathrm{a}}$ & & & $<0.001^{* * *}$ \\
\hline$\leq 0.6$ & $6(16.7)$ & $75(64.3)$ & \\
\hline$>0.6$ & $27(34.2)$ & $52(65.8)$ & \\
\hline Estradiol levels $(\mathrm{pg} / \mathrm{mL})^{\mathrm{a}}$ & & & 0.539 \\
\hline$<1000$ & $10(27)$ & $27(73)$ & \\
\hline $1000-3000$ & $18(18.4)$ & $80(81.6)$ & \\
\hline$>3000$ & $5(20)$ & $20(80)$ & \\
\hline Progesterone levels (ng/mL) ${ }^{a}$ & & & 0.941 \\
\hline$<1$ & $25(20.5)$ & $97(79.5)$ & \\
\hline$>1$ & $8(21.1)$ & 30 (78.9) & \\
\hline Grade of embryob & & & $0.007^{* *}$ \\
\hline Good $(A)$ & $16(34)$ & $31(66)$ & \\
\hline Fair (B) or Poor (C) & $17(15)$ & $96(85)$ & \\
\hline
\end{tabular}

Abbreviation: $\mathrm{AMH}$, anti-Mullerian hormone.

${ }^{\text {a }}$ Chi-square test; ${ }^{b}$ Fisher exact test; ${ }^{* * *} P<0.001$.

Table 3. Logistic Regression Analysis of Factors Affecting Clinical Pregnancy Rate

\begin{tabular}{lccc}
\hline & OR & Cl & $\boldsymbol{P}$ \\
\hline Serum AMH & 6.22 & $2.4-16.2$ & $<0.001^{* * *}$ \\
No. of embryo produced & 0.462 & $0.2-1.2$ & 0.097
\end{tabular}

Abbreviation: $\mathrm{AMH}$, anti-Mullerian hormone; OR, odds ratio.

${ }^{\mathrm{a}} \mathrm{AMH} \leq 0.6$ vs. $>0.6 ; * * * P<0.001$.

6.25, $P<0.001$, CI [2.4-16.3]) (Table 3). As expected, the number of FSH ampule was higher in women with $\mathrm{AMH}$ $\leq 0.6$ compared to those with AMH were $>0.6(P=0.002)$. In addition, endometrial thickness was higher in women with $\mathrm{AMH}>0.6$ compared to those with $\mathrm{AMH} \leq 0.6$ were $(P=0.001)$. Although we found no relationship between women's age and IVF success, further analysis showed a significant relationship between AMH level and IVF success in women $\leq 35$. (Table 4 ).

\section{Discussion}

We evaluated the role of different factors in the success of IVF/ET in infertility center of Moheb Yas hospital, Tehran, Iran. The results of our study indicate that AMH is a predictor of IVF success. Further analysis showed a significant relationship between AMH level and IVF success in women $\leq 35$, indicating that IVF success was significantly higher in younger women with higher level of $\mathrm{AMH}$ than those with a low level of AMH. The overall success rate of IVF/ET in this study was $20.6 \%$.

$\mathrm{AMH}$ is commonly used as a biomarker for evaluating the ovarian reserve. AMH serum level expresses the number of growing follicles and predicts ovarian response to stimulation (19). In a study by Cohen et al, it was shown that women with a low level of the ovarian reserve had low live birth rates after the first IVF (20). In previous studies, clinical pregnancy rate (15), live birth rates (21), the number of retrieved oocytes, and IVF fertilization rate (22) were influenced by $\mathrm{AMH}$. In a study by Silva et al, AMH levels $\leq 0.70$ accounted for $92 \%$ of the IVF
Table 4. Distribution of IVF Success by AMH Levels and Maternal Age

\begin{tabular}{llccc}
\hline \multirow{2}{*}{ Age } & & \multicolumn{2}{c}{ IVF Success } & \multirow{2}{*}{$\boldsymbol{P}$} \\
\cline { 3 - 4 } & AMH levels & Not pregnant $(\mathbf{n}=\mathbf{1 2 7})$ & Pregnant $(\mathbf{n}=\mathbf{3 3})$ & \\
\hline \multirow{2}{*}{$\leq 35$} & $>0.6$ & $25(56.8)$ & $19(43.2)$ & \multirow{2}{*}{$<0.001^{*}$} \\
& $\leq 0.6$ & $56(94.9)$ & $3(5.1)$ & \\
$>35$ & $>0.6$ & $27(77.1)$ & $8(22.9)$ & \multirow{2}{*}{0.309} \\
& $\leq 0.6$ & $19(86.4)$ & $3(13.6)$ & \\
\hline$* P<0.05$ & & &
\end{tabular}

failures (23).

Although we found a relationship between $\mathrm{AMH}$ and IVF success, we did not find any relationship between women's age and IVF success. Our result is congruent with the result of a study conducted by Verma in which no significant relationship was observed between $\mathrm{AMH}$ levels with maternal age (24). In contrast, the results of 2 studies showed that AMH was inversely correlated with women's age which means that women's higher age reduced ovarian reserve $(21,25)$. In a recent study, $\mathrm{AMH}$ could predict ovarian reserve in different age groups; however, female age was found to be a better predictor of IVF outcome than AMH (17).

We found a significant relationship between $\mathrm{AMH}$ level and IVF success in women $\leq 35$ while Goswami and Nikolaou reported that older women with higher AMH level had higher live birth rate than those with low $\mathrm{AMH}$ level (26).

We also did not find any relationship between BMI and IVF success which is in harmony with the results of a study conducted by MacKenna et al in Latin America. In the study by MacKenna which was conducted on a sample of women with a high prevalence of obesity, BMI did not influence the outcome of ART (27).

In our study, $29 \%$ of embryos had good quality and we found a relationship between the quality of blastocyst and IVF success. In a study on 350 intracytoplasmic sperm injection (ICSI) cycles, transferring one grade 1 or grade 2 blastocyst significantly increased implantation and pregnancy rates (28). The results of another study on 107 
patients undergoing IVF/ET showed that transferring two top-grade blastocysts increased implantation, pregnancy, and twinning rates to $70 \%, 87 \%$, and $61 \%$, respectively. In contrast, by transferring low-grade blastocysts, the aforementioned rates were $28 \%, 44 \%$, and $29 \%$, respectively (29).

We found no relationship between progesterone level and clinical or chemical pregnancies in women undergoing IVF. Our result was in agreement with the results of the previous study showing no significant relationship between the level of progesterone in the late follicular phase and the pregnancy rate (30). However, it is suggested that elevated progesterone level will adversely affect the endometrial environment and decrease the pregnancy rate (31). In a study, Labarta et al showed that the elevation of progesterone levels on the day of hCG injection may intervene in normal endometrial function through changes in the endometrial gene expression profile (32). The results of a systematic review conducted by Venetis et al on more than 4000 cycles showed that progesterone level on the day of hCG administration is associated with lower pregnancy rate (33).

We found no relationship between estradiol level on the day of hCG injection and IVF success. The results of a study by Foroozanfard et al indicates a positive correlation between estradiol level and IVF pregnancy rate (16). In contrast, Kondapalli et al showed that a decrease of $<10 \%$ in estradiol levels after hCG injection reduced pregnancy rate (34). Our result was in line with previous studies which found that the increased estradiol level on the day of hCG injection did not improve IVF pregnancy outcome $(35,36)$.

In our study, endometrial thickness on the day of oocyte retrieval was higher in pregnant women compared to non-pregnant women. Our results were in harmony with the findings of the previous studies which found a positive correlation between endometrial thickness and pregnancy rate $(37,38)$. Further research is needed to determine an appropriate cut-off point of endometrial thickness for good IVF outcome.

We found that women who became pregnant had produced a higher number of embryos in IVF cycles than non-pregnant did. A study conducted by Kovacs et al also showed more follicles, oocytes and therefore more embryos among pregnant women compared to nonpregnant women in IVF cycles (37).

In this study, the success rate of IVF was $20.6 \%$ which is lower compared to live birth rates of $27.1 \%$ (39), $36 \%$ (22), and $28.2 \%$ reported in previous studies (38). Considering the fact that IVF success is usually higher than the live birth rate, measures should be performed to improve the success rate of IVF in this center.

In this study, we only assessed the pregnancy rate after IVF. Since the pregnancy rate may not be the same as the live birth rate, further follow-up studies are needed to evaluate the ability of AMH to predict live birth. We also did not follow pregnancies to categorize them as normal or pathological. We recommend that in future studies longterm follow-up of IVF cycles be performed to determine the factors predicting pregnancy complications.

\section{Conclusions}

The overall success rate of IVF/ET was $20.6 \%$. AMH was an independent predictor of IVF success after adjustment for the number of embryos produced. Due to the small sample size in studies in Iran, we recommend that a meta-analysis on the factors involving in IVF success be conducted.

\section{Limitations}

A limitation of the study is its small sample size. We suggest that further studies with a larger sample size from multiple centers be conducted.

\section{Conflict of Interests}

Authors declare that they have no conflict of interests.

\section{Ethical Issues}

The Ethics Committee of Tehran University of Medical Sciences approved the research proposal. All of the recruited mothers were verbally informed about the project and were asked to fill out an informed consent form before recruitment.

\section{Financial Support}

This study received no financial support.

\section{Acknowledgement}

This study was approved by chancellery of research at Tehran University of Medical Sciences as a thesis for the fulfillment of infertility fellowship. We appreciate all women participated in the study.

\section{References}

1. Mascarenhas MN, Flaxman SR, Boerma T, Vanderpoel S, Stevens GA. National, regional, and global trends in infertility prevalence since 1990: a systematic analysis of 277 health surveys. PLoS Med. 2012;9(12):e1001356. doi:10.1371/journal.pmed.1001356

2. World Health Organization. Global prevalence of infertility, infecundity and childlessness. http://www.who.int/ reproductivehealth/topics/infertility/burden/en/.

3. Baldur-Felskov B, Kjaer SK, Albieri V, et al. Psychiatric disorders in women with fertility problems: results from a large Danish register-based cohort study. Hum Reprod. 2013;28(3):683-690. doi:10.1093/humrep/des422

4. Davari Tanha F, Mohseni M, Ghajarzadeh M. Sexual function in women with primary and secondary infertility in comparison with controls. Int J Impot Res. 2014;26(4):132134. doi:10.1038/ijir.2013.51

5. Kjaer TK, Jensen A, Dalton SO, Johansen C, Schmiedel S, Kjaer SK. Suicide in Danish women evaluated for fertility problems. Hum Reprod. 2011;26(9):2401-2407. 
doi:10.1093/humrep/der188

6. Kamel RM. Assisted reproductive technology after the birth of louise brown. J Reprod Infertil. 2013;14(3):96-109.

7. Gleicher N, Weghofer A, Barad D. Update on the comparison of assisted reproduction outcomes between Europe and the USA: the 2002 data. Fertil Steril. 2007;87(6):1301-1305. doi:10.1016/j.fertnstert.2006.11.024

8. Traub ML, Van Arsdale A, Pal L, Jindal S, Santoro N. Endometrial thickness, Caucasian ethnicity, and age predict clinical pregnancy following fresh blastocyst embryo transfer: a retrospective cohort. Reprod Biol Endocrinol. 2009;7:33. doi:10.1186/1477-7827-7-33

9. van Loendersloot LL, van Wely M, Limpens J, Bossuyt PM, Repping S, van der Veen F. Predictive factors in in vitro fertilization (IVF): a systematic review and meta-analysis. Hum Reprod Update. 2010;16(6):577-589. doi:10.1093/ humupd/dmq015

10. Thomas MR, Sparks AE, Ryan GL, Van Voorhis BJ. Clinical predictors of human blastocyst formation and pregnancy after extended embryo culture and transfer. Fertil Steril. 2010;94(2):543-548. doi:10.1016/j.fertnstert.2009.03.051

11. Firns S, Cruzat VF, Keane KN, et al. The effect of cigarette smoking, alcohol consumption and fruit and vegetable consumption on IVF outcomes: a review and presentation of original data. Reprod Biol Endocrinol. 2015;13:134. doi:10.1186/s12958-015-0133-X

12. Pandian Z, Bhattacharya S, Ozturk O, Serour G, Templeton A. Number of embryos for transfer following in-vitro fertilisation or intra-cytoplasmic sperm injection. Cochrane Database Syst Rev. 2009(2):Cd003416. doi:10.1002/14651858.CD003416.pub3

13. Nelson SM, Lawlor DA. Predicting live birth, preterm delivery, and low birth weight in infants born from in vitro fertilisation: a prospective study of 144,018 treatment cycles. PLoS Med. 2011;8(1):e1000386. doi:10.1371/journal. pmed.1000386

14. Tremayne S, Akhondi MM. Conceiving IVF in Iran. Reprod Biomed Soc Online. 2016;2:62-70. doi:10.1016/j. rbms.2016.07.002

15. Ashrafi M, Hemat M, Arabipoor A, Salman Yazdi R, Bahman-Abadi A, Cheraghi R. Predictive values of antimullerian hormone, antral follicle count and ovarian response prediction index (ORPI) for assisted reproductive technology outcomes. J Obstet Gynaecol. 2017;37(1):82-88. doi:10.1080/01443615.2016.1225025

16. Foroozanfard F, Moraveji SA, Taghavi SA, Karimi F. Association Between Serum Estradiol Level on the Day of hCG Administration and IVF-ICSI Outcome. J Obstet Gynaecol India. 2016;66(3):170-173. doi:10.1007/s13224015-0687-8

17. Shahrokh Tehraninezhad E, Mehrabi F, Taati R, Kalantar V, Aziminekoo E, Tarafdari A. Analysis of ovarian reserve markers (AMH, FSH, AFC) in different age strata in IVF/ ICSI patients. Int J Reprod Biomed (Yazd). 2016;14(8):501506.

18. Heitmann RJ, Hill MJ, Richter KS, DeCherney AH, Widra EA. The simplified SART embryo scoring system is highly correlated to implantation and live birth in single blastocyst transfers. J Assist Reprod Genet. 2013;30(4):563-567. doi:10.1007/s10815-013-9932-1

19. Fleming R, Seifer DB, Frattarelli JL, Ruman J. Assessing ovarian response: antral follicle count versus anti-Mullerian hormone. Reprod Biomed Online. 2015;31(4):486-496. doi:10.1016/j.rbmo.2015.06.015

20. Cohen J, Mounsambote L, Prier P, et al. Outcomes of first IVF/ICSI in young women with diminished ovarian reserve. Minerva Ginecol. 2017;69(4):315-321. doi:10.23736/s00264784.16.04003-X

21. Keane K, Cruzat VF, Wagle S, Chaudhary N, Newsholme $\mathrm{P}$, Yovich J. Specific ranges of anti-Mullerian hormone and antral follicle count correlate to provide a prognostic indicator for IVF outcome. Reprod Biol. 2017;17(1):51-59. doi:10.1016/j.repbio.2016.12.002

22. Bolat SE, Ozdemirci S, Kasapoglu T, Duran B, Goktas L, Karahanoglu E. The effect of serum and follicular fluid antiMullerian hormone level on the number of oocytes retrieved and rate of fertilization and clinical pregnancy. North Clin Istanb. 2016;3(2):90-96. doi:10.14744/nci.2016.02418

23. Silva JB, Panaino TR, Tamm MA, et al. Prediction of metaphase II oocytes according to different serum AntiMullerian hormone (AMH) levels in antagonist ICSI cycles. JBRA Assist Reprod. 2016;20(4):222-226. doi:10.5935/15180557.20160043

24. Verma AK, Rajbhar S, Mishra J, et al. Anti-Mullerian Hormone: A Marker of Ovarian Reserve and its Association with Polycystic Ovarian Syndrome. J Clin Diagn Res. 2016;10(12):Qc10-qc12. doi:10.7860/jcdr/2016/20370.8988

25. Barbakadze L, Kristasashvili J. Antimullerian hormone in cases of different reproductive pathologies. Georgian Med News. 2014(232-233):16-21.

26. Goswami M, Nikolaou D. Is AMH Level, Independent of Age, a Predictor of Live Birth in IVF? J Hum Reprod Sci. 2017;10(1):24-30. doi:10.4103/jhrs.JHRS_86_16

27. MacKenna A, Schwarze JE, Crosby JA, Zegers-Hochschild F. Outcome of assisted reproductive technology in overweight and obese women. JBRA Assist Reprod. 2017;21(2):79-83. doi:10.5935/1518-0557.20170020

28. Balaban B, Urman B, Sertac A, Alatas C, Aksoy S, Mercan R. Blastocyst quality affects the success of blastocyststage embryo transfer. Fertil Steril. 2000;74(2):282-287. doi:10.1016/S0015-0282(00)00645-2

29. Gardner DK, Lane M, Stevens J, Schlenker T, Schoolcraft WB. Blastocyst score affects implantation and pregnancy outcome: towards a single blastocyst transfer. Fertil Steril. 2000;73(6):1155-1158. doi:10.1016/S0015-0282(00)005185

30. Yding Andersen C, Bungum L, Nyboe Andersen A, Humaidan P. Preovulatory progesterone concentration associates significantly to follicle number and $\mathrm{LH}$ concentration but not to pregnancy rate. Reprod Biomed Online. 2011;23(2):187-195. doi:10.1016/j. rbmo.2011.04.003

31. Huang B, Ren X, Wu L, et al. Elevated Progesterone Levels on the Day of Oocyte Maturation May Affect Top Quality Embryo IVF Cycles. PLoS One. 2016;11(1):e0145895. doi:10.1371/journal.pone.0145895

32. Labarta E, Martinez-Conejero JA, Alama $\mathrm{P}$, et al. Endometrial receptivity is affected in women with high circulating progesterone levels at the end of the follicular phase: a functional genomics analysis. Hum Reprod. 2011;26(7):1813-1825. doi:10.1093/humrep/der126

33. Venetis CA, Kolibianakis EM, Bosdou JK, Tarlatzis BC. Progesterone elevation and probability of pregnancy after IVF: a systematic review and meta-analysis of over 60 000 cycles. Hum Reprod Update. 2013;19(5):433-457. doi:10.1093/humupd/dmt014 
34. Kondapalli LA, Molinaro TA, Sammel MD, Dokras A. A decrease in serum estradiol levels after human chorionic gonadotrophin administration predicts significantly lower clinical pregnancy and live birth rates in in vitro fertilization cycles. Hum Reprod. 2012;27(9):2690-2697. doi:10.1093/ humrep/des 216

35. Wu Z, Li R, Ma Y, et al. Effect of HCG-day serum progesterone and oestradiol concentrations on pregnancy outcomes in GnRH agonist cycles. Reprod Biomed Online. 2012;24(5):511-520. doi:10.1016/j.rbmo.2012.02.003

36. Yu Ng EH, Yeung WS, Yee Lan Lau E, So WW, Ho PC. High serum oestradiol concentrations in fresh IVF cycles do not impair implantation and pregnancy rates in subsequent frozen-thawed embryo transfer cycles. Hum Reprod. 2000;15(2):250-255.

37. Kovacs P, Matyas S, Boda K, Kaali SG. The effect of endometrial thickness on IVF/ICSI outcome. Hum Reprod. 2003;18(11):2337-2341. doi:10.1093/humrep/deg461

38. Vaegter KK, Lakic TG, Olovsson M, Berglund L, Brodin T, Holte J. Which factors are most predictive for live birth after in vitro fertilization and intracytoplasmic sperm injection (IVF/ICSI) treatments? Analysis of 100 prospectively recorded variables in $8,400 \mathrm{IVF} / \mathrm{ICSI}$ singleembryo transfers. Fertil Steril. 2017;107(3):641-648.e642. doi:10.1016/j.fertnstert.2016.12.005

39. Rhenman A, Berglund L, Brodin T, et al. Which set of embryo variables is most predictive for live birth? A prospective study in 6252 single embryo transfers to construct an embryo score for the ranking and selection of embryos. Hum Reprod. 2015;30(1):28-36. doi:10.1093/ humrep/deu295

(C) 2018 The Author (s); This is an open-access article distributed under the terms of the Creative Commons Attribution License (http://creativecommons.org/licenses/by/4.0), which permits unrestricted use, distribution, and reproduction in any medium, provided the original work is properly cited. 